This document is the Accepted Manuscript version of a Published Work that appeared in final form in Analytical Chemistry, copyright (c) American Chemical Society after peer review and technical editing by the publisher. To access the final edited and published work see:

https://dx.doi.org/10.1021/acs. analchem.9b04369. 


\title{
Lab in a Tube: Point-of-Care detection of Escherichia coli
}

\author{
Niloufar Amin ${ }^{\mathrm{a}, \mathrm{b}, \mathrm{c}, \$}$, Amadeo Sena Torralba ${ }^{\mathrm{a},}$, Ruslan Álvarez-Diduk $^{\mathrm{a}}$, Abbas Afkhami ${ }^{\mathrm{b}}$ and Arben \\ Merkoçi ${ }^{\mathrm{a}, \mathrm{d}^{*}}$ \\ ${ }^{a}$ Nanobioelectronics \& Biosensors Group, Catalan Institute of Nanoscience and Nanotechnology (ICN2), CSIC and BIST, \\ Campus UAB, Bellaterra, 08193 Barcelona, Spain. \\ ${ }^{b}$ Faculty of Chemistry, Bu-Ali Sina University, Hamedan, Iran \\ ${ }^{c}$ Food and Drug Laboratory Research Center, Food and Drug Organization, MOH\&ME, Tehran, Iran \\ ${ }^{d}$ ICREA, Institució Catalana de Recerca i Estudis Avançats, Pg. Lluís Companys 23, 08010 Barcelona, Spain. \\ $\ddagger$ These authors contributed equally to the work. \\ *arben.merkoci@icn2.cat
}

ABSTRACT: Significant levels of infectious diseases caused by pathogenic bacteria are nowadays a worldwide concern implying considerable public healthcare challenges and huge economic detriment. Due to the fast spread of these bio-threat agents and the outbreak of diseases, a rapid detection of pathogens at early stages is crucial, particularly in low-resource settings. To this aim, we developed for the first time a new sensing approach carried out in a single-step for Escherichia coli O157:H7 detection. The detection principle is based on Förster Resonance Energy Transfer using gold nanoclusters as signal reporter and gold nanoparticles conjugated with antibodies as a quencher. The sensing platform includes and UV-LED to provide the proper excitation and consists in a microtube containing two pieces of fiber glass; one of them is embedded with label-free gold nanoclusters and the other one with gold nanoparticles conjugated with antibodies. Upon the addition of the sample containing bacteria the florescence of gold nanoclusters is recovered. The assay was evaluated by naked eye (on/off) and quantitatively using a smartphone camera. The biosensor proved to be highly specific and sensitive, achieving a limit of detection as low as $4.0 \mathrm{CFU} \mathrm{ml}{ }^{-1}$. Additionally, recoveries of $110 \%$ and $95 \%$ were obtained when evaluating the platform in spiked river and tap water respectively.

Keywords: Point-of-Care; Biosensor; Optical detection; Bacteria detection; FRET; Smartphone-based detection

Nowadays, Escherichia coli O157:H7 (E. coli O157:H7) is one of the major threats to public healthcare causing serious infectious diseases mainly through contaminated water and food ${ }^{1,2}$. Since the causative pathogen agent can spread rapidly, it is imperative to achieve fast diagnosis. In addition, providing a quick decision in the field to test the safety of water and food will have a great effect on controlling and minimizing mortality rate and financial affliction.

Traditional methods for the identification and detection of pathogenic bacteria are mainly based on plate cultivation ${ }^{3}$, enzymelinked immunosorbent assay (ELISA) and polymerase chain reaction $(\mathrm{PCR})^{4-6}$. Culturing method requires long analysis time $(\sim$ days) and special labor operation. PCR and ELISA need significant instruments, manpower, expense and time that already are not suitable for emergency cases especially in developing countries where medical facilities are unobtainable ${ }^{7}$.

To overcome the aforementioned problems, point-of-care $(\mathrm{PoC}) \mathrm{di}$ agnostic can be a potential solution for improving the global health managements ${ }^{8}$. Toward this important goal, different PoC devices for detection of $E$. coli have been implemented including colorimetric-based lateral flow immunoassay (LFIA) ${ }^{9-11}$ and other nonpaper-based microfluidic devices based on electrochemical detection ${ }^{12}$, colorimetry ${ }^{13,}{ }^{14}$, fluorimetry ${ }^{15}$ and magneto-fluorimetry ${ }^{16}$. LFIA are limited by their poor sensitivity that usually requires signal amplification, while the other microfluidic systems are relatively expensive and require trained personnel ${ }^{17,18}$. Therefore, there is a lack of a suitable PoC device to detect of E. coli for in field assays.

Our research group have been involved in developing photoluminescence paper-based platforms for detecting $E$. coli ${ }^{19,20}$. The reported strategies are related to nanoswitch "Off-On" methodology using photoluminescent quantum dots conjugated with antibodies. In these platforms, despite of being sensitive and specific, there are some drawbacks such as multistep procedures, exclusive readers platforms and particularly the easy to use for end-user that reduce the applicability of these biosensors for in-field measurements.

Previous studies have reported the quenching of gold nanoclusters (AuNCs) fluorescence in the presence of gold nanoparticles (AuNPs) ${ }^{21}$. This phenomenon occurs due to the complementary overlapping between the surface plasmon resonance absorption wavelength of AuNp and the fluorescence emission wavelength of AuNCs. Some biosensors have been developed by exploiting this phenomenon such as the detection of cholesterol ${ }^{22}$ and uric acid ${ }^{23}$. In these cases, the detection is carried out through the production of $\mathrm{H}_{2} \mathrm{O}_{2}$ in an enzymatic reaction, AuNPs seeds start growing, increasing the absorbance of AuNPs with the consequently decrease in the fluorescence emission of AuNCs. Although this strategy has been exploited for biosensing applications, so far, to the best of our knowledge none of these nanomaterials have being used together as an immunosensor. Specifically, if we are starting from the OFF state, fluorescence could be recovered if the distance between the donor/acceptor pair becomes big enough

Certainly E. coli bacteria is big enough to promote this effect. For this purpose, we developed a simple, fast and cost-effective "lab on a tube" platform for $E$. coli detection. The proposed approach relies on Förster Resonance Energy Transfer (FRET) mechanism based 
on gold nanoclusters (AuNCs) as energy donor and antibody-functionalized AuNPs as energy acceptor. This enables an ON/OFF fluorescent signal depending on the presence or absence of E. coli in the sample. The assay is carried out inside a microtube that contains the reagents embedded in glass fiber membrane. In this regard, the biosensor can be easily transported and stored up to use. The assay starts by just introducing the sample into the micro-tube. After 20 minutes of incubation, the result can be evaluated by naked eye, in which the presence or absence of fluorescence indicates a yes/no response to the bacteria. Conveniently, it can be evaluated quantitatively by using a 3D printed device with an UV-LED, a smartphone camera and an image-analysis app.

\section{EXPERIMENTAL SECTION}

Materials and Instruments. All commercial reagents are analytical grade and handled according to the material safety data sheets suggested by the suppliers. Hydrogen tetrachloroaurate (III) dehydrate (HAuCl4 3H2O, 99.9\%), Trisodium Citrate, Sodium hydroxide, Phosphate buffered saline tablet (P4417), bovine serum albumin (BSA), were purchased from Sigma-Aldrich (Madrid, Spain). Anti-E.coli antibody (Ab, ab68451) was obtained from Abcam (Cambridge, UK). Escherichia coli O157:H7 (CECT 4783, E. coli) and Salmonella Typhimurium (CECT 722T, S. typhimurium) strains were obtained from Sigma-Aldrich (Madrid, Spain). Analytical grade nitric acid and hydrochloric acid were purchased from Fisher Scientific Fiber glass membrane (GFCP00080000) was purchased from Millipore. All glassware were washed with aqua regia, and rinsed three times with Milli-Q water. Transmission electron microscopy (TEM) images were performed with a FEI Tecnai G2 20 TWIN electron microscope. Fluorescence emission and Absorbance spectra were collected on a Microplate reader (Molecular device). TS-100 Thermo-Shaker (Biosan, Riga, Latvia) was used as the stirrer for functionalization of AuNPs with antibodies.

Synthesis of BSA-AuNCs. BSA-AuNCs were synthesized according to a previous protocol ${ }^{24}$. Briefly, aqueous solutions of $\mathrm{HAuCl} 4$ $(10 \mathrm{mM}, 5 \mathrm{ml})$ and BSA $(50 \mathrm{mg} / \mathrm{ml}, 5 \mathrm{ml})$ were mixed and subjected to vigorous stirring at $37^{\circ} \mathrm{C}$. After $2 \mathrm{~min}, \mathrm{NaOH}$ solution $(1.0$ $\mathrm{M}, 0.5 \mathrm{~mL}$ ) was added into the reaction mixture. The reaction container was sealed and allowed to proceed in the dark for $12 \mathrm{~h}$. The solution became deep brown and showed strong red emission under a 365-nm UV lamp, pointing out the formation of the AuNCs. The prepared AuNCs were purified through dialysis membrane (MWCO: $1 \mathrm{kDa}$ ) against Milli-Q water for one day to remove unreacted $\mathrm{HAuCl}_{4}$ and sodium hydroxide. Purified AuNCs were kept at $4{ }^{\circ} \mathrm{C}$ for further use. The concentration of BSA-AuNCs was estimated at around $20 \mathrm{mg} / \mathrm{ml}$.

Synthesis and conjugation of AuNPs. The synthesis of AuNPs was performed following the protocol reported by Bastús and coworkers ${ }^{25}$. In short, the initial AuNPs seeds were prepared by boiling $150 \mathrm{~mL}$ of sodium citrate $(2.2 \mathrm{mM})$ in a sealed condenser, followed by the injection of $1 \mathrm{ml}$ of $\mathrm{HAuCl}_{4}(25 \mathrm{mM})$. After 10 minutes, the color of the solution turned from light yellow to pink and it was cooled up to $90^{\circ} \mathrm{C}$. Next, $1 \mathrm{ml}$ of sodium citrate $(60 \mathrm{mM})$ was added and after $2 \mathrm{~min}, 1 \mathrm{ml}$ of $\mathrm{HAuCl}_{4}(25 \mathrm{mM})$ was sequentially added. The solution was kept under stirring at $90{ }^{\circ} \mathrm{C}$ for 30 min and the color of solution finally became wine-red. The final concentration of the AuNPs solution was $3.1 \times 10^{11} \mathrm{NPs} / \mathrm{ml}$.

The AuNPs were bioconjugated with antibodies against E. coli following the procedure previously reported by our group ${ }^{26}$. First the $\mathrm{pH}$ of the AuNPs was adjusted to 8.9 using Borate buffer $(10 \mathrm{mM})$. Next, $100 \mu \mathrm{L}$ of anti-E. coli $(200 \mu \mathrm{g} / \mathrm{mL})$ were incubated with 1.5 $\mathrm{ml}$ AuNPs for $24 \mathrm{~h}$ at $350 \mathrm{rpm}$ and $4{ }^{\circ} \mathrm{C}$. Then $100 \mu \mathrm{L} \mathrm{BSA}$ solution in Milli-Q water (final concentration $1 \%(\mathrm{w} / \mathrm{w})$ ) was added to the conjugated AuNPs followed by an incubation for $1 \mathrm{~h}$ under the previous conditions. The excess of antibodies and BSA were removed by centrifuging the solution at $14000 \mathrm{rpm}$ and $4{ }^{\circ} \mathrm{C}$ for $30 \mathrm{~min}$, followed by re-suspension with $200 \mu \mathrm{L}$ of Milli-Q water. Finally the conjugated AuNPs where stored in $4^{\circ} \mathrm{C}$ for further use.

Platform fabrication. The glass fiber membranes were cut into a $15 \mathrm{~cm} \times 8 \mathrm{~mm}$ strips. $800 \mu \mathrm{L}$ of the conjugated AuNPs and AuNCs where drop-casted on separate glass fiber strips and dried in a vacuum chamber for 2.5 hours. Finally they were cut in circles (diameter $\approx 0.6 \mathrm{~cm}$ ) using a hole puncher and introduced in the microtubes.

Bacteria preparation. Stock solution $(1010$ E. coli $/ \mathrm{ml})$ of bacteria was prepared by dissolving $10 \mathrm{mg}$ E. coli in $1 \mathrm{ml}$ filtered Milli Q water followed by shaking with vortex. In order to determine the concentration of the prepared stock solution, the optical density at $600 \mathrm{~nm}$ (OD600) was measured using a spectrophotometer and corroborated with Agilent geometry software (https://www.chem.agilent.com/store/biocalculators/calcODBacte rial.jsp). Standard serial solutions of E. coli $\left(0-10^{8} \mathrm{CFU} / \mathrm{ml}\right)$ were prepared by diluting the suspension stock solution $(1010 \mathrm{CFU} / \mathrm{ml}$ $\approx 1010$ E. coli $/ \mathrm{ml}$ ) in PBS.

Assay performance. Serial dilutions of E. coli $\left(0-10^{8} \mathrm{CFU} / \mathrm{ml}\right)$ where prepared in PBS, $200 \mu \mathrm{L}$ where drop-casted in the microtube and incubated for $20 \mathrm{~min}$ in room temperature. The assay was evaluated with a spectrophotometer by transferring the solution into a microplate. The fluorescence intensity was measured at $665 \mathrm{~nm}$ (Ext. $365 \mathrm{~nm}$ ) for each dilution of $E$. coli and normalized with the black $\left(\mathrm{F}-\mathrm{F}_{0}\right)$, where $\mathrm{F}$ and $\mathrm{F}_{0}$ corresponded to sample with and without $E$. coli, respectively. For a point-of-care application, the assay was evaluated by taking a picture using a mobile phone camera and analyzing it with ImageJ software (National Institutes of Health, Maryland, USA) following the protocol already reported by our group $^{27}$. All the experiments were replicated three times.

\section{RESULTS AND DISCUSSION}

Assay principle. The assay was performed in a polypropylene microtube, which was used as a reservoir and detection platform. Firstly, the fluorescent AuNCs were synthesized, purified and drop-casted on a fiberglass membrane. The fiber glass was then dried using a vacuum desiccator and cut into circular pieces $(\varnothing 0.6$ $\mathrm{cm}$ ) using a hole puncher. The pieces were introduced into the microtube and stored until running the assay. The same procedure was done for the AuNPs, which were previously conjugated with antibodies against $E$. coli $\mathrm{O} 157: \mathrm{H} 7$ following the procedure reported in the experimental section (Figure 1A). In this way, the micro-tube stocked both conjugate pads, one containing the sensing PL probe (AuNCs) and the other containing the quencher label (AuNPs). The fiber glass was used as a porous material with high absorption and release capacity of solutions, appropriate long-term storage of samples, high stability and good flexibility ${ }^{28,29}$. In order to evaluate the presence of $E$. coli in a real environment, the assay starts by introducing the sample solution into the microtube followed by a 20 minutes incubation step (Figure 1B). The sample solution enables the rehydration of the conjugate pad and the eventual release of the stored nanomaterials to the solution. The biosensing mechanism is based on Förster Resonance Energy Transfer (FRET), being the AuNPs and AuNCs the energy acceptors and donors, respectively. In this regard, the AuNPs were able to quench the fluorescence emission of the AuNCs when they were close together. This is known as the "turn-off" mode, which is linked to the absence of $E$. coli in the sample. Conversely, in the presence of E. coli, the 
antibody-functionalized AuNPs is attached to the surface of the bacteria avoiding the approach of the AuNCs. In this way, due to the fact that FRET mechanism is possible up to $30 \mathrm{~nm}$ and E. coli where $1 \mu \mathrm{m}$ sized ${ }^{30}$, the fluorescence signal was restored, switching to a "turn-on" mode (Figure 1C). It should be noticed the specificity of the assay. In this regard, AuNCs were mixed with AuNPs with and without bacteria. It can be observed in Figure S1 that there is no recovery in the fluorescence of AuNCs when AuNPs are not conjugated with antibodies.

Finally, a quantitative outcome was obtained using a smartphone camera. Nowadays, almost any smartphone has a good quality CMOS sensor and the possibility to fix the picture parameters such as shutter speed, ISO and focus, obtaining reproducible and accurate images. In this regard, a 3D printed device was developed to introduce the micro-tube using the cap as the sample holder. The device contains a $365 \mathrm{~nm}$ UV-LED to excite the AuNCs and a micro lens to adjust the focal length of the camera to the minimum possible (Figure 1D). The pictures were analyzed using ImageJ app (smartphone version) to transform the brightness levels into a numerical value, which allows quantification. Since most of the people across the world has a smartphone as a common equipment in our day-to-day life, this proof of concept makes a highly promising device for point-of-care assay and field applications without the need to a conventional bulky analyzer such as fluorimeter.

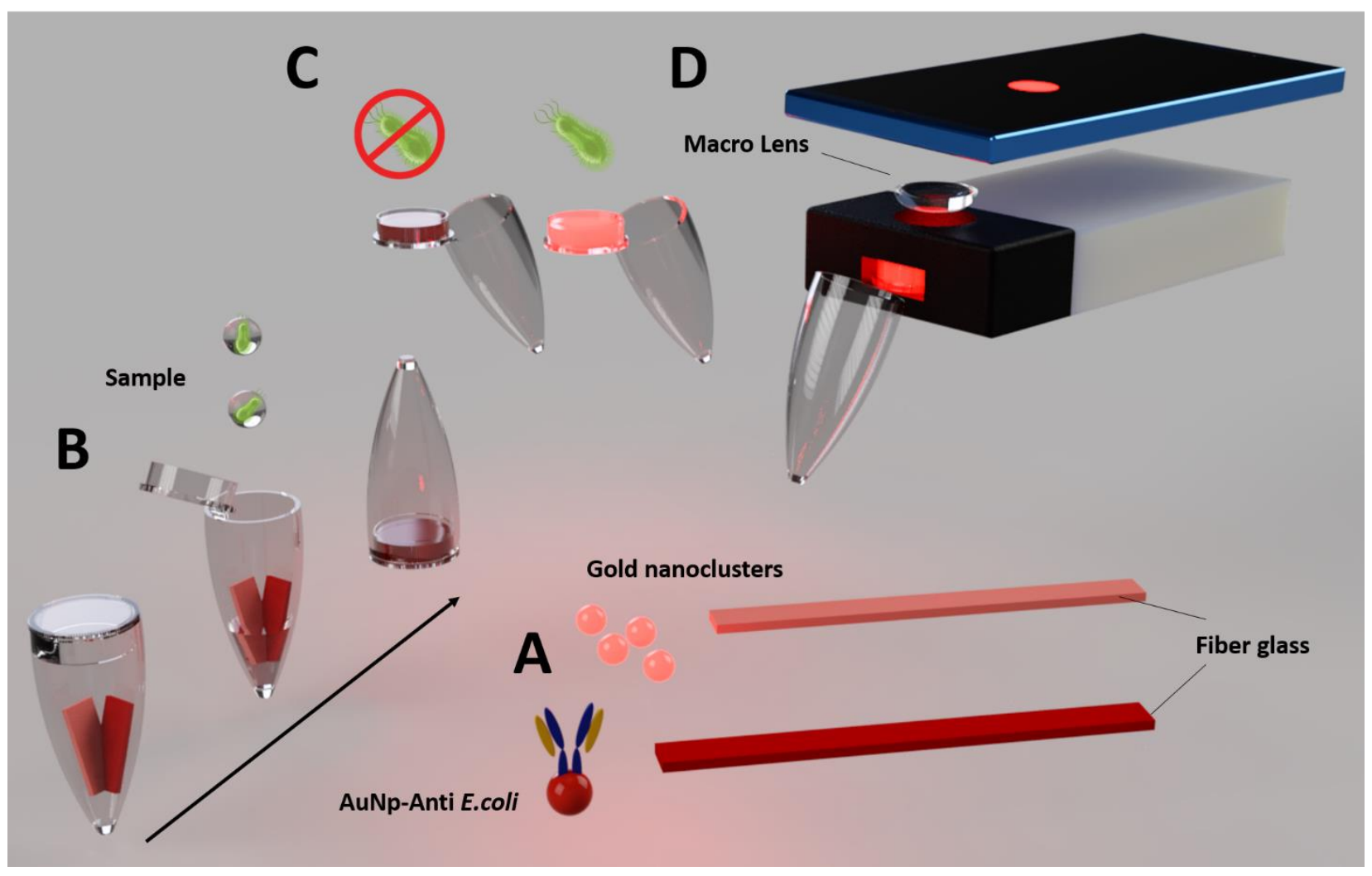

Figure 1. Schematic representation of the biosensing platform. (A) Two fiber glass strips embedded with AuNCs and AuNPs conjugated with anti-E. coli antibody respectively. (B) Operation principle; Sample is added directly to the micro-tube containing the two strips. (C) Naked eye evaluation of the ON/OFF switch mechanism. (D) Smartphone based device for quantitative evaluation of the E. coli concentration.

Characterization of AuNCs and conjugated-AuNPs. The synthesized AuNCs were characterized by transmission electron microscopy (TEM). As observed in Figure 2A, they have a uniform morphology and a narrow size distribution with an average diameter of about $1.0 \mathrm{~nm}$. Furthermore, a fluorescence emission peak at $665 \mathrm{~nm}$ when excited at $365 \mathrm{~nm}$ and a strong red emission under UV light (Figure 2B). The synthesized AuNPs were also characterized with TEM, as shown in Figure 2C and Figure 2D. AuNPs has a spherical and uniform size of around $40 \mathrm{~nm}$. Besides, its conjugation to antibodies against $E$. coli was corroborated by the presence of red shift from 525 to $535 \mathrm{~nm}$ in the UV-vis spectrum, indicating a modification on the AuNPs surface (Figure S2) ${ }^{31}$. Finally, in order to achieve FRET, the absorbance spectrum of energy acceptor must be overlapped with the emission spectrum of the donor probe. This phenomenon can be observed in Figure 2B, where the emission spectrum of the AuNCs was clearly overlapped with the absorbance spectrum of the conjugated-AuNPs. 

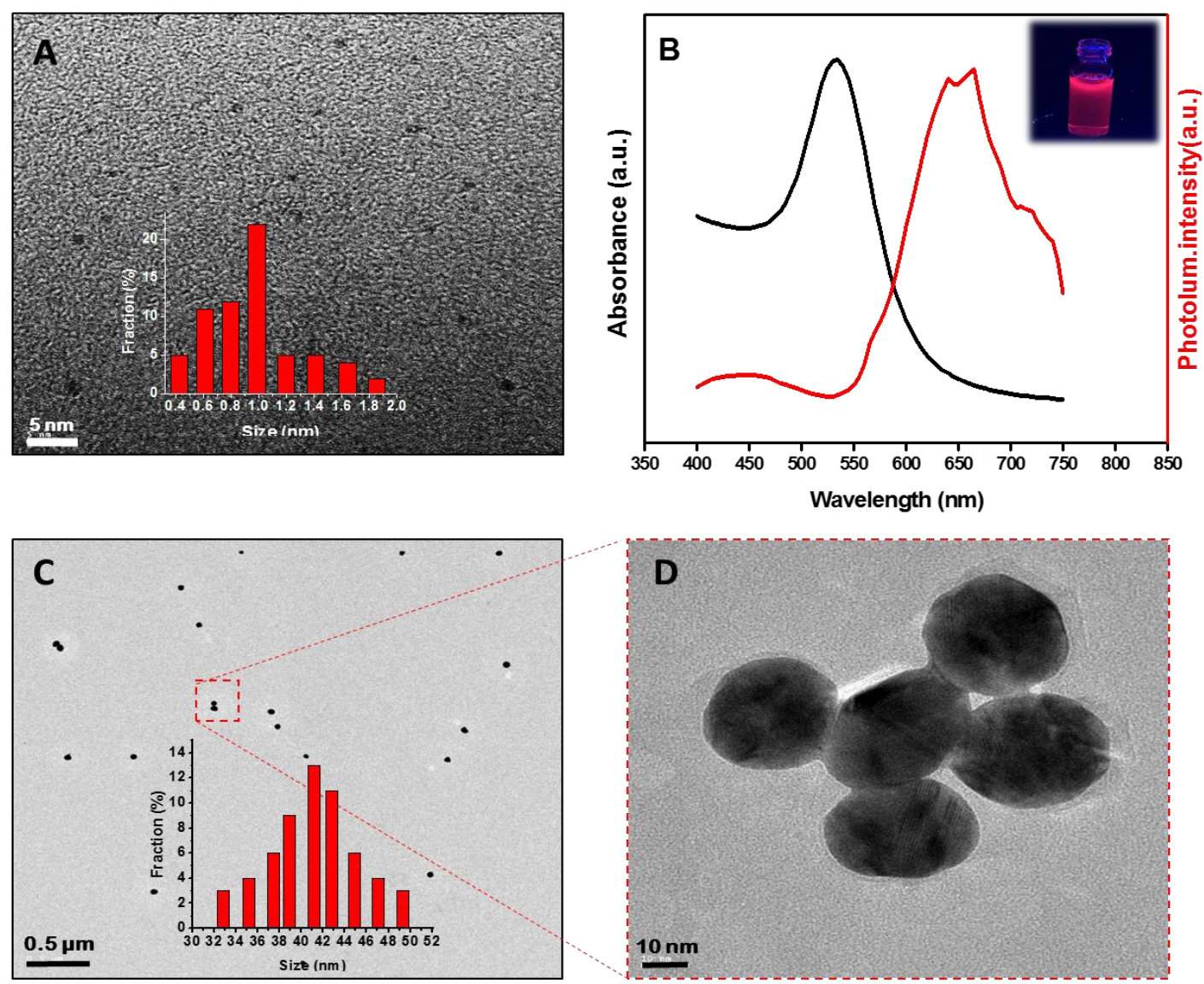

Figure 2. (A) TEM image of BSA-AuNCs. Inset: the size distribtion histogram of the as prepared AuNCs. (B) Fluorescence spectra of BSAAuNCs (red line) and absorption spectrum of conjugated AuNPs with Anti-E. coli (black line). Inset: Digital photo of AuNCs under UV light. (C) TEM image of Citrate stabilized- AuNPs. Inset: graph corresponds to size distribution diagram. (D) High magnified of as-prepared AuNPs.

Assay optimization. The highest concentration with the higher fluorescence emission of AuNCs was tested to evaluate the size dependence on the quenching efficiency of AuNPs. It is known that, the bigger the size of nanoparticles, the highest the red shift in the wavelength, causing more overlap with the emission of AuNCs ${ }^{21}$. Among the tested sizes, $40 \mathrm{~nm}$ was selected as the most suitable for the assay (Figure S3). Besides bigger nanoparticles $(>40 \mathrm{~nm})$ produce more quenching, the antibodies conjugation protocols are fully optimized for $40 \mathrm{~nm}$. Moreover, if bigger nanoparticles are used the required amount of antibodies to cover the surface will be higher, causing a more expensive device. To evaluate how the AuNP concentration affects the quenching of AuNCs, the stock solution $\left(3.1 \times 10^{11} \mathrm{NPs} / \mathrm{ml}\right)$ of AuNP was concentrated (by centrifugation) and diluted. It can be observed in Figure 3A that indeed higher concentrations of AuNP produces more quenching on the light emission of AuNCs. Nevertheless, we have selected 1.16 $\times 10^{12}$ $\mathrm{NP} / \mathrm{ml}$ as a compromise between efficiency and affordability since there is no noteworthy quenching at higher concentrations.

Furthermore, the antibody concentration on the AuNPs was also optimized as it dramatically affects the sensitivity and the cost-effectiveness of the platform (using less antibody can considerably reduce the development cost of the biosensor). This was done by performing the well-stablished gold aggregation test (GAT) ${ }^{32}$, which determines the maximum concentration of antibody required to cover the AuNPs surface. Based on the results shown in Figure 3B, we concluded that $175 \mu \mathrm{g} / \mathrm{ml}$ of anti-E. coli were enough to cover the surface of the AuNPs, however we round it up to 200 $\mu \mathrm{g} / \mathrm{ml}$ in the subsequent assays.

The required concentration of AuNCs to achieve an effective FRET and recovery was another parameter to be optimized. In this context, (AuNP at fixed concentration of $1.16 \times 10^{12} \mathrm{NP} / \mathrm{ml}$ ) the fluorescence recovery in presence of $E$. coli was evaluated using different AuNCs concentrations (from 0.5 to $8.0 \mathrm{mg} / \mathrm{ml}$ ). As expected, an excess of AuNCs brought to signal saturation while a low concentration of AuNCs let to negligible fluorescence intensity (Figure 3C). Therefore, the fluorescence enhancement efficiency $\left(\mathrm{F}-\mathrm{F}_{0}\right)$ was employed as a criterion to evaluate the best signal where $\mathrm{F}$ and $\mathrm{F}_{0}$ correspond to the intensities of fluorescence of AuNCs/AuNPs system in the presence and absence of E. coli, respectively. Hereby, $6.0 \mathrm{mg} / \mathrm{ml}$ was determined as the optimal concentration of AuNCs. 

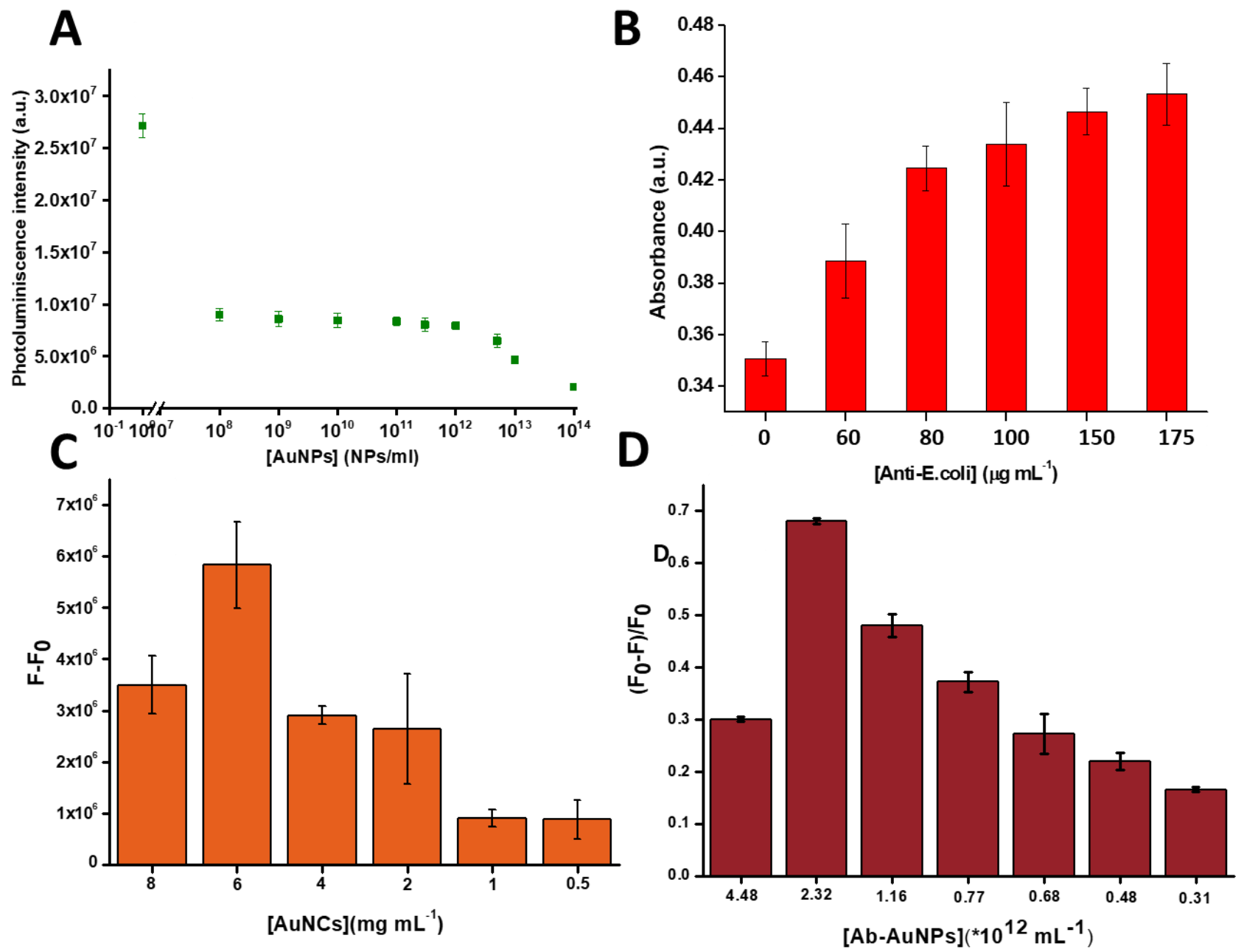

Figure 3. Optimization of experimental conditions. (A) Pre-optimization of the quenching efficiency due to AuNPs concentration (B) The effect of the concentration of the immobilized antibody on the surface of AuNPs (GAT) at NaCl $1 \%$ and $0.58 \times 10^{12} / \mathrm{ml}$ AuNPs in various concentration of antibody (C) Effect of the concentration of AuNCs as a fluorophore agent on fluorescence recovery efficiency $\left(\mathrm{F}-\mathrm{F}_{0}\right)$ where $\mathrm{F}$ and $\mathrm{F}_{0}$ correspond to the fluorescence intensities of AuNCs/AuNPs system in the presence and absence of $E$. coli, respectively at $10^{5}$ $\mathrm{CFU} / \mathrm{mL}$ E. coli (D) The effect of the concentration of anti-E. coli- labeled AuNPs on quenching efficiency $\left(\mathrm{F}_{0}-\mathrm{F}\right) / \mathrm{F}_{0}$ where $\mathrm{F}$ and $\mathrm{F}_{0}$ correspond to the fluorescence intensities of AuNCs in the presence and absence of Ab-AuNPs, respectively. The concentration of AuNCs was $6.0 \mathrm{mg} / \mathrm{ml}$.

Although AuNPs concentration was previously optimized (pre optimization strategy) those nanoparticles were not conjugated with anti-E. coli antibodies. It is important to double check the concentration of conjugated AuNPs required to achieve an effective FRET taking in consideration the previously optimized AuNCs concentration. In this regard, conjugated AuNPs ranging from 0.31 to 4.46 $\left(\mathrm{x} 10^{12} \mathrm{NPs} / \mathrm{mL}\right)$ were evaluated. As observed in Figure 3D, 2.32 $\left(\mathrm{x} 10^{12}\right.$ AuNPs $\left./ \mathrm{mL}\right)$ allowed a $68 \%$ quenching efficiency, while higher and lower concentrations ended up with signal saturation and low quenching, respectively. It is also essential to optimize this parameter for fluorescence restoration efficiency (Figure S4). Therefore, this was the concentration of conjugated AuNPs selected for the next experiments.

Finally, in order to better understand the response rate of our platform to $E$. coli detection, a comprehensive kinetic study was performed (Figure S5) showing that the fluorescence signal increased exponentially and reached equilibrium after 20 minutes. This reveals the assay time required before performing the final evaluation.

Smartphone-based detection of E. coli. The developed platform was firstly evaluated under optimal conditions by spiking known concentrations of $E$. coli $\left(0\right.$ to $\left.10^{7} \mathrm{CFU} / \mathrm{mL}\right)$ in PBS buffer $(10 \mathrm{mM}$ $\mathrm{pH}$ 7.4) and using a spectrophotometer. As observed in Figure 4A, the fluorescence signal gradually increases with higher concentration of $E$. coli, with a good correlation (exponential behavior $\log [E$. coli]) in the range of 0 to $10^{5} \mathrm{CFU} / \mathrm{mL}$ (Figure $4 \mathrm{~B}$ ), being $\mathrm{F}$ and $\mathrm{F}_{0}$ the fluorescence intensities at $665 \mathrm{~nm}$ in the presence and absence of E. coli, respectively. The limit of detection (LOD) was calculated as low as $1.37 \mathrm{CFU} / \mathrm{mL}$ based on 3 times "s/m" ratio, where "s" is the standard deviation of the blank fluorescence signal (three replicates) and " $\mathrm{m}$ " is the slope of the related calibration curve. Furthermore, the assay was evaluated with a smartphone camera, in order to get as close as possible to point-of-care conditions. In this 
context, a Samsung galaxy S7 smartphone was used to take a picture of the microtubes after running the assay (20 minutes incubation). The micro-tube cap was irradiated under a UV LED (Ex. 365 $\mathrm{nm}$ ) and the smartphone was attached to a holder, the pictures were always taken in the same conditions; at ISO 100 and shooter speed $1 / 60 \mathrm{~s}$. As illustrated in Figure 4C, the sample containing 0 $\mathrm{CFU} / \mathrm{mL}$ of $E$. coli, defined as blank sample, is characterized by low brightness levels due to the "turn-off" FRET effect. However, with the increase of $E$. coli concentration there was a gradual increase in fluorescence ("turn-on" mode). In order to obtain a numerical value, the pictures were analyzed with Image $J$ software, in which the fluorescence intensity is transformed into a value from 0 to 255 (where 0 is dark and 255 is maximum brightness). The fluorescence intensity values were normalized with the signal corre- sponding to absence of $E$. coli $\left(\mathrm{F}_{0}\right)$. The calibration curve is observed in Figure 4D, which shows a power fluorescence increase . It is worth to notice a change in the mathematical function in light of the results obtained with the fluorimeter and the smartphone. Graphing the values according to the function obtained with the fluorometer $(\log [E$. coli $])$, it can be seen an exponential behavior which means that the results obtained with the smartphone follows a power function from 0 to $10^{7} \mathrm{CFU} / \mathrm{mL}$ with a LOD of 4.0 $\mathrm{CFU} / \mathrm{mL}$. (Figure 4D inset)

The developed biosensor performance of $E$. coli detection was compared to some of the reported point of care sensors and summarized in Table S1.
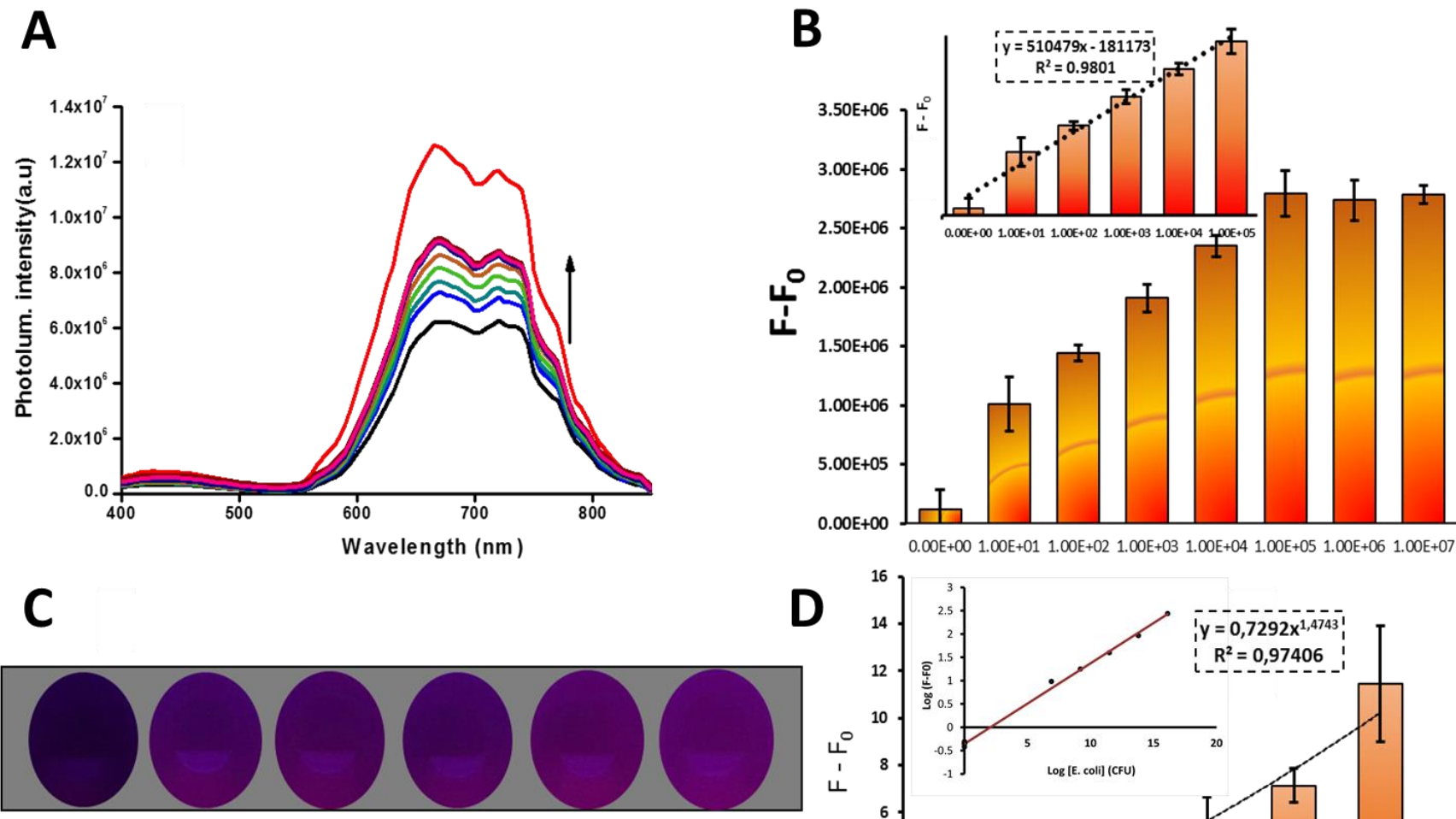

Blank $10^{3} \mathrm{CFU} \mathrm{mL}^{-1}$ $10^{7} \mathrm{CFU} \mathrm{mL}^{-1}$

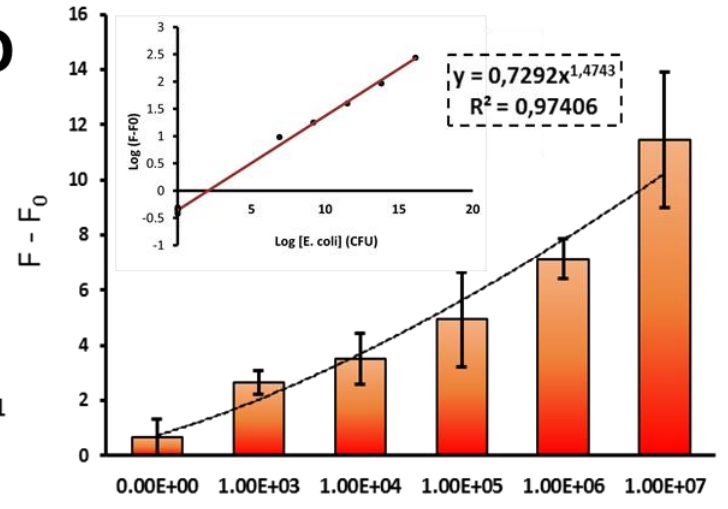

[E. coli], (CFU mL-1)

Figure 4. Quantification of E. coli O157:H7 by developed biosensor. (A) Fluorescence spectrum of AuNCs/Ab-AuNPs upon addition of different concentrations of $E$. coli from 0 to $10^{7} \mathrm{CFU} / \mathrm{mL}$. (B) Calibration curve of the assay by using fluorimeter. (F and $\mathrm{F}_{0}$ correspond to the fluorescence intensities of AuNCs/AuNPs system in the presence and absence of E. coli, respectively). (C) Typical images of AuNCs/AbAuNPs system in the presence of various concentration of $E$. coli in the range of $0-10^{7} \mathrm{CFU} / \mathrm{mL}\left(0,10^{3}, 10^{4}, 10^{5}, 10^{6} \mathrm{CFU} / \mathrm{mL}\right.$ from left to right) captured by smartphone camera under $365 \mathrm{~nm}$ UV light irradiation; (D) Calibration curve resulted by processing images in the mobile phone. Error bars represent the standard deviation $(n=3)$ inset: linear behavior of a power fuction. 
Real samples evaluation. To further test the practicability of the biosensor, the assay was conducted by spiking known concentrations of E. coli $\left(10^{2}\right.$ and $\left.10^{5} \mathrm{CFU}\right)$ to tap water and river water samples collected from river Ter (Vic, Spain). The samples were evaluated following the procedure previously reported and the recoveries where calculated by comparing the results obtained in real samples (i) with the ones obtained in PBS (ii), using the following equation:

$$
\text { Recovery }(\%)=100 \times(F-F 0)^{i} /(F-F 0)^{i i}
$$

The results are summarized in Table 1. The obtained recoveries prove that there is no drastic interference when evaluating samples with a complex matrix, thus the developed platform can be applied for the detection of $E$. coli in real samples at the point-of-care.

Table.1. Detection of $E$. coli bacteria spiked in water samples $(\mathrm{n}=3)$

\begin{tabular}{|l|l|l|l|l|}
\hline Sample & $\begin{array}{l}\text { Spiked } \\
\text { E.coli } \\
\left(\text { CFUml }^{-1}\right)\end{array}$ & $\begin{array}{l}\text { \#F-F0 in } \\
\text { standard } \\
\text { buffer }\end{array}$ & $\begin{array}{l}\text { *F-F }_{0} \text { in } \\
\text { real sample }\end{array}$ & $\begin{array}{l}\text { Recovery } \\
(\%)^{\mathrm{a}}\end{array}$ \\
\hline \multirow{2}{*}{ River } & $10^{2}$ & 1440843 & 1503611 & 103.70 \\
water & $10^{5}$ & 2791463 & 2648975 & 105.37 \\
\cline { 2 - 5 } & $10^{2}$ & 1440843 & 1400975 & 97.23 \\
Tap & $10^{5}$ & 2783852 & 2740975 & 98.45 \\
water & & & & \\
\hline
\end{tabular}

${ }^{\mathrm{a}}$ Recovery $(\%)=100 \times\left(\mathrm{F}-\mathrm{F}_{0}{ }^{*} / \mathrm{F}^{-\mathrm{F}_{0}}{ }^{\#}\right)$

Determination of the assays specificity. The specificity of the biosensor toward E. coli was assessed by evaluation samples containing E.coli and Salmonella typhimurium, which is a similar family pathogen. Dilutions of E. coli, Salmonella and a mixture of both bacteria where spiked in PBS. The assay was carried out following the previous procedure. As shown in Figure 5, the normalized fluorescence (FL) intensity increased with higher concentrations of $E$. coli $\left(0\right.$ to $\left.10^{5} \mathrm{CFU} / \mathrm{mL}\right)$. Conversely, the normalized FL intensity obtained for a sample with $10^{5} \mathrm{CFU} / \mathrm{mL}$ of Salmonella was similar to the one obtained for $0 \mathrm{CFU}$ of $E$. coli. This result prove that the developed biosensor was only specific for $E$. coli due to the AuNPs functionalization with highly specific antibodies against $E$. coli O157:H7. This was further corroborated by evaluating solutions containing a mixture of $10^{5} \mathrm{CFU} / \mathrm{mL}$ Salmonella $+10^{2} \mathrm{CFU} / \mathrm{mL} E$. coli and $10^{5} \mathrm{CFU} / \mathrm{mL}$ E. coli $+10^{2} \mathrm{CFU} / \mathrm{mL}$ Salmonella. In the first case, the obtained signal was similar to the blank $(0 \mathrm{CFU} / \mathrm{mL} E$. coli) and in the second case the signal was similar to the one obtained for $10^{5} \mathrm{CFU} / \mathrm{mL}$ E. coli. Therefore, the results confirmed that the platform has a high specificity response and when applied in real samples, the achieved signal could be linked only to $E$. coli detection.

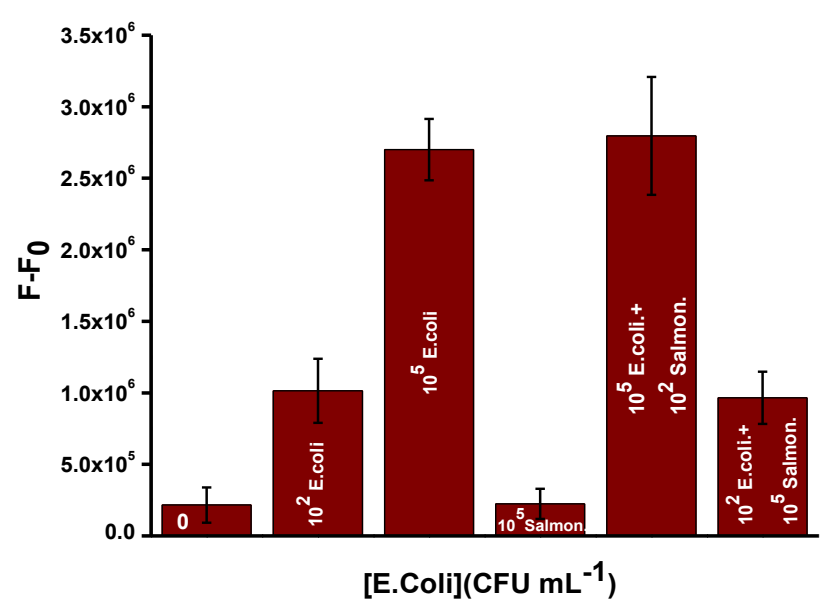

Figure 5. Specificity of the developed fluorescence biosensor toward E. coli in the presence of Salmonella as interfering bacteria. The error bars are the standard deviation of the three measurements.

\section{CONCLUSIONS}

The proposed all-in-one device combines outstanding advantage of both the glass fiber membrane as a cost-effective substrate with high loading capacity and the microtube platform as simple and portable set-up. Moreover, the detection method applied in the developed biosensor is fast (20 minutes) and doesn't require washing steps. It has proved to be highly sensitive and specific for detection of $E$. coli, although its application can be extended to other big analytes. Finally, the possibility to do smartphone-based detection empowers the developed biosensor as unique PoC tests with real applicability in resource limited settings.

\section{ASSOCIATED CONTENT}

\section{Supporting Information}

UV-vis spectra of AuNPs and Ab-AuNPs; Effect of concentration of Ab-AuNPs on the photoluminescence restoration; Kinetic behavior studies of AuNCs/Ab-AuNPs in the presence of E. coli.

\section{AUTHOR INFORMATION}

\section{* Corresponding author}

E-mail address: arben.merkoci@icn2.cat

Note:

The authors declare no competing financial interest.

\section{ACKNOWLEDGMENTS}

The ICN2 is funded by the CERCA Programme / Generalitat de Catalunya. The ICN2 is supported by the Severo Ochoa program of the Spanish Ministry of Economy, Industry and Competitiveness (MINECO, grant No. SEV-2017-0706). Financial support was obtained under MINECO project MAT2017-87202-P. N.A. acknowledges Food and Drug Laboratory Research Center, Food and Drug Organization of Iran and Ministry of Science, Research and Technology of Iran for scholarship during this research time. A.S.T. acknowledges Universitat Autònoma de Barcelona for the 
possibility of carrying out this work inside the framework of its $\mathrm{PhD}$ Programme in Biotechnology.

\section{REFERENCES}

1.Painter, J. A.; Hoekstra, R. M.; Ayers, T.; Tauxe, R. V.; Braden, C. R.; Angulo, F. J.; Griffin, P. M., Attribution of foodborne illnesses, hospitalizations, and deaths to food commodities by using outbreak data, United States, 1998-2008. Emerging Infect. Dis. 2013, 19 (3), 407.

2.Scallan, E.; Griffin, P. M.; Angulo, F. J.; Tauxe, R. V.; Hoekstra, R. M., Foodborne illness acquired in the United Statesunspecified agents. Emerging Infect. Dis. 2011, 17 (1), 16.

3.Opota, O.; Croxatto, A.; Prod'hom, G.; Greub, G., Blood culturebased diagnosis of bacteraemia: state of the art. Clin. Microbiol. Infect. 2015, 21 (4), 313-322.

4.Souza, R. B.; Trevisol, D. J.; Schuelter-Trevisol, F., Bacterial sensitivity to fosfomycin in pregnant women with urinary infection. Braz. J. Infect. Dis. 2015, 19 (3), 319-323.

5.Zourob, M.; Elwary, S.; Turner, A. P., Principles of bacterial detection: biosensors, recognition receptors and microsystems. Springer Science \& Business Media: 2008.

6.Zhao, Z.; Yan, R.; Yi, X.; Li, J.; Rao, J.; Guo, Z.; Yang, Y.; Li, W.; Li, Y.-Q.; Chen, C., Bacteria-activated theranostic nanoprobes against methicillin-resistant Staphylococcus aureus infection. ACS nano 2017, 11 (5), 4428-4438.

7.Russo, L.; Leva Bueno, J.; Bergua, J. F.; Costantini, M.; Giannetto, M.; Puntes, V.; de la Escosura-Muñiz, A.; Merkoçi, A. Low-cost strategy for the development of a rapid electrochemical assay for bacteria detection based on $\mathrm{AuAg}$ nanoshells. ACS Omega 2018, 3 (12), 18849-18856.

8.Yetisen, A. K.; Akram, M. S.; Lowe, C. R., based microfluidic point-of-care diagnostic devices. Lab Chip 2013, 13 (12), 22102251

9.Huang, H.; Zhao, G.; Dou, W., Portable and quantitative pointof-care monitoring of Escherichia coli O157: H7 using a personal glucose meter based on immunochromatographic assay. Biosens. Bioelectron. 2018, 107, 266-271.

10.Luo, K.; Hu, L.; Guo, Q.; Wu, C.; Wu, S.; Liu, D.; Xiong, Y.; Lai, W., Comparison of 4 label-based immunochromatographic assays for the detection of Escherichia coli O157: H7 in milk. $J$. Dairy Sci. 2017, 100 (7), 5176-5187.

11.Wu, W.; Zhao, S.; Mao, Y.; Fang, Z.; Lu, X.; Zeng, L., A sensitive lateral flow biosensor for Escherichia coli O157: H7 detection based on aptamer mediated strand displacement amplification. Anal. Chim. Acta 2015, 861, 62-68.

12.Li, Y.; Xiong, Y.; Fang, L.; Jiang, L.; Huang, H.; Deng, J.; Liang, W.; Zheng, J., An electrochemical strategy using multifunctional nanoconjugates for efficient simultaneous detection of Escherichia coli O157: H7 and Vibrio cholerae O1. Theranostics 2017, 7 (4), 935.

13.Chen, J.; Alcaine, S. D.; Jackson, A. A.; Rotello, V. M.; Nugen, S. R., Development of engineered bacteriophages for Escherichia coli detection and high-throughput antibiotic resistance determination. ACS Sens. 2017, 2 (4), 484-489.

14.Lagally, E.; Scherer, J.; Blazej, R.; Toriello, N.; Diep, B.; Ramchandani, M.; Sensabaugh, G.; Riley, L.; Mathies, R., Integrated portable genetic analysis microsystem for pathogen/infectious disease detection. Anal. chem. 2004, 76 (11), $3162-3170$

15.Sugino, H.; Ozaki, K.; Shirasaki, Y.; Arakawa, T.; Shoji, S.; Funatsu, T., On-chip microfluidic sorting with fluorescence spectrum detection and multiway separation. Lab Chip 2009, 9 (9), 1254-1260.
16.Banerjee, T.; Sulthana, S.; Shelby, T.; Heckert, B.; Jewell, J.; Woody, K.; Karimnia, V.; McAfee, J.; Santra, S., Multiparametric magneto-fluorescent nanosensors for the ultrasensitive detection of Escherichia coli O157: H7. ACS Infect. Dis. 2016, 2 (10), 667-673. 17.Zarei, M., Infectious pathogens meet point-of-care diagnostics. Biosens. Bioelectron. 2018, 106, 193-203.

18.Nasseri, B.; Soleimani, N.; Rabiee, N.; Kalbasi, A.; Karimi, M.; Hamblin, M. R., Point-of-care microfluidic devices for pathogen detection. Biosens. and Bioelectron. 2018, 117, 112-128.

19.Cheeveewattanagul, N.; Morales-Narváez, E.; Hassan, A. R. H.; Bergua, J. F.; Surareungchai, W.; Somasundrum, M.; Merkoçi, A., Straightforward immunosensing platform based on graphene oxide-decorated nanopaper: a highly sensitive and fast biosensing approach. Adv. Funct. Mater. 2017, 27 (38), 1702741.

20.Morales-Narváez, E.; Naghdi, T.; Zor, E.; Merkoçi, A., Photoluminescent lateral-flow immunoassay revealed by graphene oxide: highly sensitive paper-based pathogen detection. Anal. chem. 2015, 87 (16), 8573-8577.

21.Qin, H.; Ma, D.; Du, J., Distance dependent fluorescence quenching and enhancement of gold nanoclusters by gold nanoparticles. Spectrochim. Acta A 2018, 189, 161-166.

22.Chang, H.-C.; Ho, J.-a. A., Gold nanocluster-assisted fluorescent detection for hydrogen peroxide and cholesterol based on the inner filter effect of gold nanoparticles. Anal. chem. 2015, 87 (20), 10362-10367.

23.Liu, Y.; Li, H.; Guo, B.; Wei, L.; Chen, B.; Zhang, Y., Gold nanoclusters as switch-off fluorescent probe for detection of uric acid based on the inner filter effect of hydrogen peroxide-mediated enlargement of gold nanoparticles. Biosens. Bioelectron. 2017, 91, 734-740.

24.Xie, J.; Zheng, Y.; Ying, J. Y., Protein-directed synthesis of highly fluorescent gold nanoclusters. J. Am. Chem. Soc. 2009, 131 (3), 888-889.

25.Bastús, N. G.; Comenge, J.; Puntes, V., Kinetically controlled seeded growth synthesis of citrate-stabilized gold nanoparticles of up to $200 \mathrm{~nm}$ : size focusing versus Ostwald ripening. Langmuir 2011, 27 (17), 11098-11105.

26.Haiss, W.; Thanh, N. T.; Aveyard, J.; Fernig, D. G., Determination of size and concentration of gold nanoparticles from UV- Vis spectra. Anal. chem. 2007, 79 (11), 4215-4221.

27.Álvarez-Diduk, R.; Orozco, J.; Merkoçi, A., Paper stripembedded graphene quantum dots: a screening device with a smartphone readout. Sci. Rep. 2017, 7 (1), 976.

28.Oyama, Y.; Osaki, T.; Kamiya, K.; Kawano, R.; Honjoh, T.; Shibata, H.; Ide, T.; Takeuchi, S., A glass fiber sheet-based electroosmotic lateral flow immunoassay for point-of-care testing. Lab Chip 2012, 12 (24), 5155-5159.

29.O'Farrell, B., Evolution in lateral flow-based immunoassay systems. In Lateral flow immunoassay, Springer: 2009; pp 1-33.

30.Morales-Narváez, E.; Hassan, A. R.; Merkoçi, A., Graphene Oxide as a Pathogen-Revealing Agent: Sensing with a Digital-Like Response. Angew. Chem. 2013, 52 (51), 13779-13783.

31.Chen, W.; Cao, F.; Zheng, W.; Tian, Y.; Xianyu, Y.; Xu, P.; Zhang, W.; Wang, Z.; Deng, K.; Jiang, X., Detection of the nanomolar level of total Cr [(III) and (VI)] by functionalized gold nanoparticles and a smartphone with the assistance of theoretical calculation models. Nanoscale 2015, 7 (5), 2042-2049.

32.Ambrosi, A.; Castañeda, M. T.; Killard, A. J.; Smyth, M. R.; Alegret, S.; Merkoçi, A., Double-codified gold nanolabels for enhanced immunoanalysis. Anal. chem. 2007, 79 (14), 5232-5240. 
Graphical Table of Contents

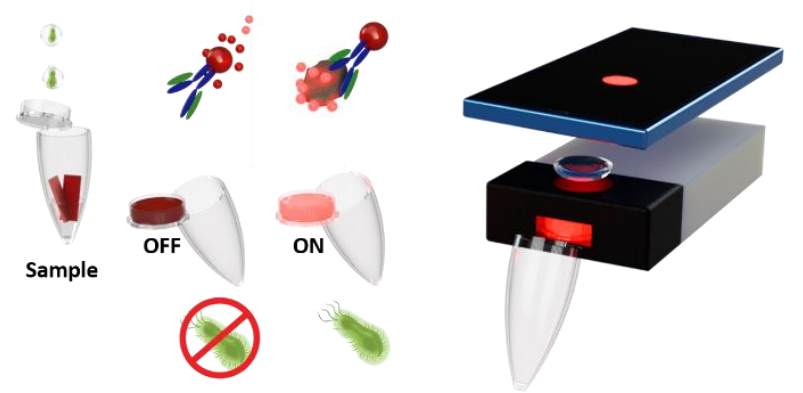

